\title{
Transcavernous Resection of a Giant Extensive Chondrosarcoma with Endoscopic Assistance
}

\author{
Walid Elshamy $^{1,2} \quad$ Burcak Soylemez $^{1} \quad$ Sima Sayyahmelli $^{1} \quad$ Nese Keser $^{1} \quad$ Mustafa K. Baskaya $^{1}$ \\ ${ }^{1}$ Department of Neurological Surgery, University of Wisconsin- \\ Madison School of Medicine and Public Health, Madison, Wisconsin, \\ United States \\ 2 Department of Neurological Surgery, Ain Shams University, Faculty \\ of Medicine, Cairo, Egypt \\ Address for correspondence Mustafa K. Baskaya, MD, Department of \\ Neurological Surgery, University of Wisconsin-Madison, 600 Highland \\ Avenue, CSC K8/828, Madison, WI 53792, United States \\ (e-mail: m.baskaya@neurosurgery.wisc.edu).
}

J Neurol Surg B Skull Base 2022;83(suppl S3):e644-e645.

\begin{abstract}
Keywords

- chondrosarcoma

- endoscopic-assisted microsurgery

- cavernous sinus

- skull base

- transcavernous approach

Chondrosarcomas are one of the major malignant neoplasms which occur at the skull base. These tumors are locally invasive. Gross total resection of chondrosarcomas is associated with longer progression-free survival rates. The patient is a 55-year-old man with a history of dysphagia, left eye dryness, hearing loss, and left-sided facial pain. Magnetic resonance imaging (MRI) showed a giant heterogeneously enhancing leftsided skull base mass within the cavernous sinus and the petrous apex with extension into the sphenoid bone, clivus, and the cerebellopontine angle, with associated displacement of the brainstem (-Fig. 1). An endoscopic endonasal biopsy revealed a grade-II chondrosarcoma. The patient was then referred for surgical resection. Computed tomography (CT) scan and CT angiogram of the head and neck showed a left-sided skull base mass, partial destruction of the petrous apex, and complete or near-complete occlusion of the left internal carotid artery. Digital subtraction angiography confirmed complete occlusion of the left internal carotid artery with cortical, vertebrobasilar, and leptomeningeal collateral development. The decision was made to proceed with a left-sided transcavernous approach with possible petrous apex drilling. During surgery, minimal petrous apex drilling was necessary due to autopetrosectomy by the tumor. Endoscopy was used to assist achieving gross total resection (-Fig. 2). Surgery and postoperative course were uneventful. MRI confirmed gross total resection of the tumor. The histopathology was a grade-II chondrosarcoma. The patient received proton therapy and continues to do well without recurrence at 4-year follow-up. This video demonstrates steps of the combined microsurgical skull base approaches for resection of these challenging tumors.

The link to the video can be found at: https://youtu.be/WImCP_-i57s.
\end{abstract}

received

April 30, 2020

accepted after revision

January 9, 2021

published online

May 11, 2021 www.thieme.com/skullbasevideos

www.thieme.com/jnlsbvideos
DOI https://doi.org/

$10.1055 / \mathrm{s}-0041-1727124$ ISSN 2193-6331.

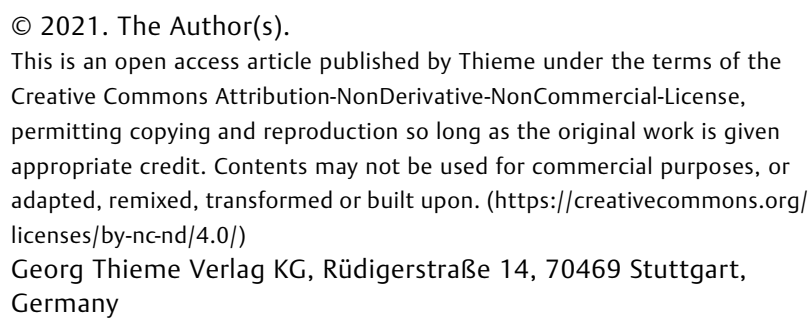




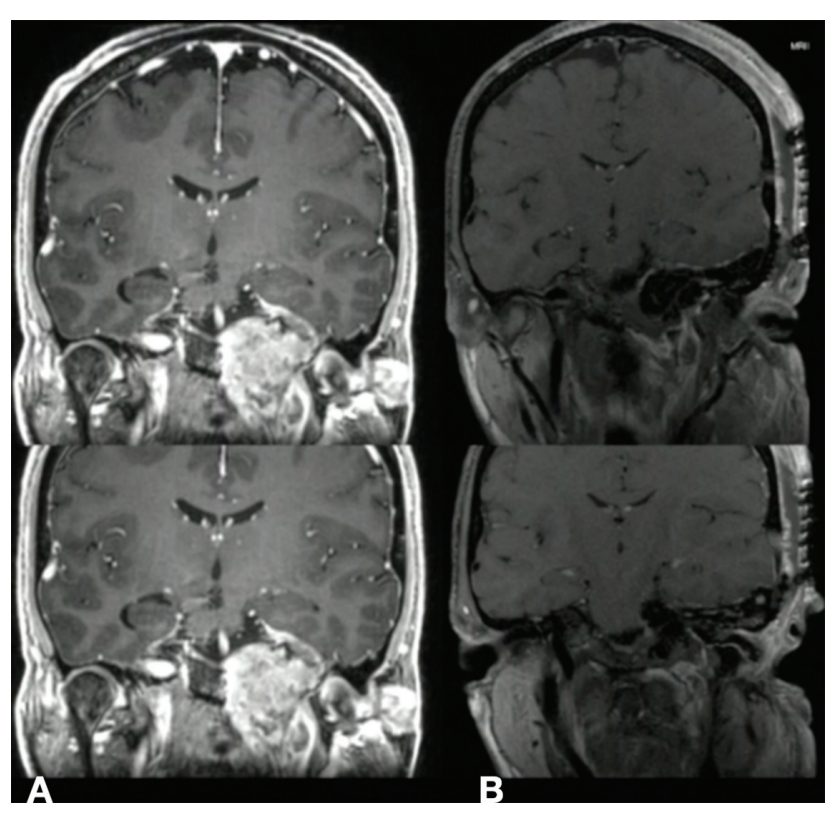

Fig. 1 (A) Preoperative coronal T1-weighted MRI with contrast shows heterogeneously enhancing lesion in the cavernous sinus extending to the posterior fossa, sphenoid sinus infratemporal fossa. (B) Postoperative T1-weighted MRI with contrast shows gross total resection of the lesion from the middle fossa, infratemporal fossa and cavernous sinus. MRI, magnetic resonance imaging.

\section{Note}

This manuscript has not been published before, either as a whole or in part, nor is it under consideration for publication elsewhere. Additionally, the authors of this manuscript report no conflicts of interest related to the manuscript, which was not financially supported by any person or institute.

\section{Disclosure}

The authors have no personal, financial, or institutional interest in any of the drugs, materials, or devices described in this article.

\section{Conflict of Interest}

None declared.

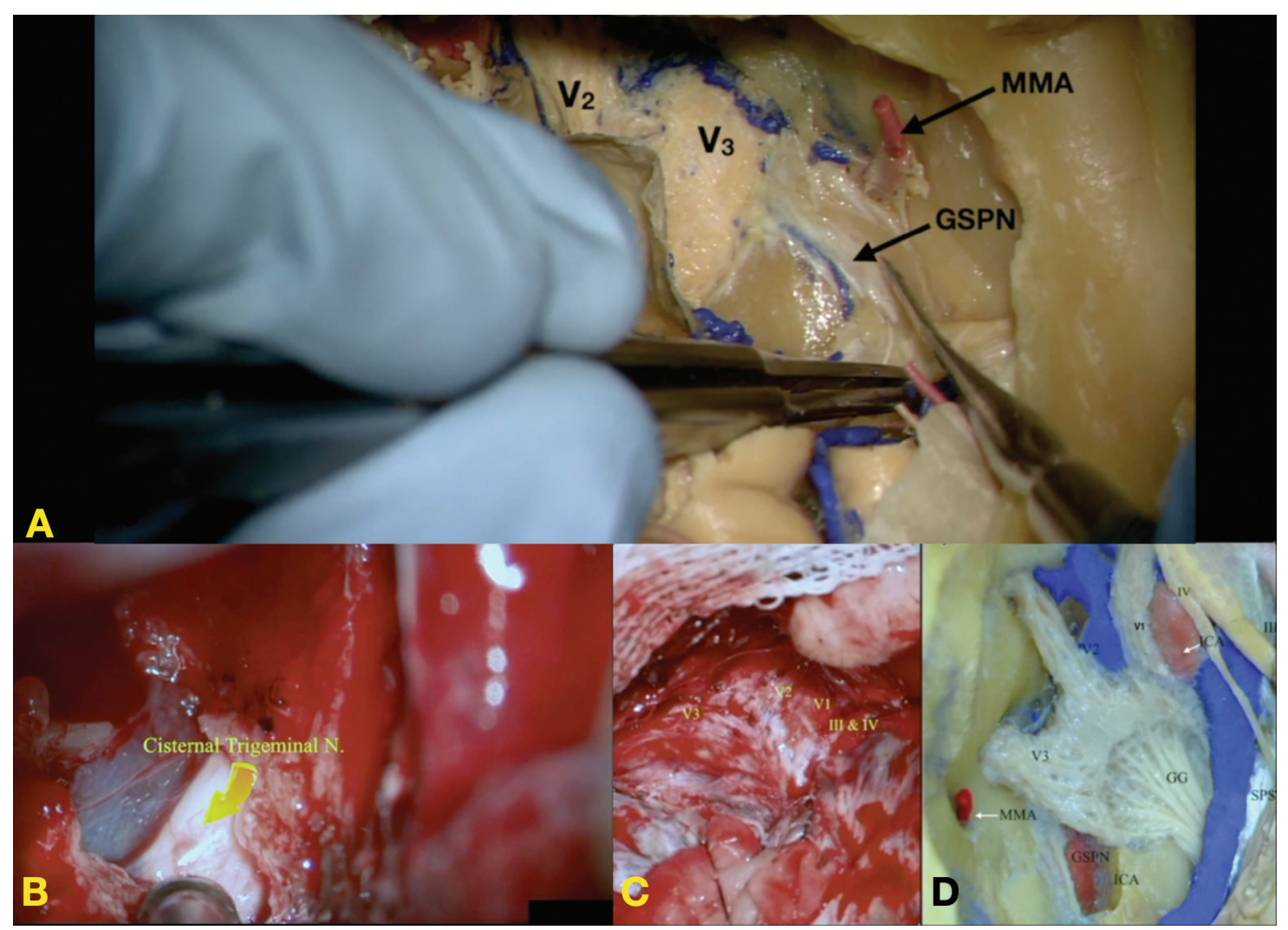

Fig. 2 Cadaveric and intraoperative photomicrographs. (A) Cadaveric dissection of the cavernous sinus and the middle cranial fossa showing middle meningeal artery (MMA), V1, V2 branches of the trigeminal nerve, and greater superficial petrosal nerve (GSPN). (B) Intraoperative image shows the cisternal segment of the trigeminal nerve after sectioning of the posterior fossa dura and tentorium and removal of the tumor. (C) Intraoperative image shows the cavernous sinus tumor splaying the branches of the trigeminal nerve including V1, V2, and V3. (D) A cadaveric dissection of the cavernous sinus showing the branches of the trigeminal nerve (V1, V2, V3), Gasserian ganglion (GG), trochlear nerve (IV), oculomotor nerve (III), and cavernous internal carotid artery (ICA) after drilling of the overlying bone, middle meningeal artery (MMA), the superior petrosal sinus (SPS), and the greater superficial petrosal nerve (GSPN). 